\title{
The effects of thiamine pyrophosphate on propofol-induced oxidative liver injury and effect on dysfunction
}

\author{
Leman Acun Delen ${ }^{1}$, Zeliha Korkmaz Dişli², Hakan G. Taş3 , Ufuk Kuyrukluyildiz ${ }^{4}$, \\ Gulce N. Yazici ${ }^{5}$, Bahadir Süleyman ${ }^{6}$, Mehmet Kuzucu${ }^{7}$, Cebrail Gürsu' ${ }^{8}$ and Halis Suleyman ${ }^{6}$ \\ ${ }^{1}$ Department of Anesthesiology and Reanimation, Malatya Training and Research Hospital, Malatya, Turkey \\ 2 Department of Reanimation, Inönü University Turgut Ozal Medicine Center, Malatya, Turkey \\ ${ }^{3}$ Siran State Hospital, Gümüşhane, Turkey \\ ${ }^{4}$ Department of Anesthesiology and Reanimation, Faculty of Medicine, Erzincan Binali Yildirim University, Erzincan, \\ Turkey \\ ${ }^{5}$ Department of Histology, Faculty of Medicine, Erzincan Binali Yildirim University, Erzincan, Turkey \\ ${ }^{6}$ Department of Pharmacology, Faculty of Medicine, Erzincan Binali Yildirim University, Erzincan, Turkey \\ ${ }^{7}$ Faculty of Science and Literature, Erzincan Binali Yildirim University, Erzincan, Turkey \\ ${ }^{8}$ Department of Physiology, Faculty of Medicine, Erzincan Binali Yildirim University, Erzincan, Turkey
}

\begin{abstract}
Propofol may cause an increase in reactive oxygen species in the body. In this study, we tested the effect of antioxidant thiamine pyrophosphate (TPP) on propofol-induced liver damage. The eighteen rats were split into three groups: $\mathrm{HG}$, healthy; PP, propofol-treated $(50 \mathrm{mg} / \mathrm{kg})$ and PT, treated with propofol ( $50 \mathrm{mg} / \mathrm{kg})$ and TPP $(25 \mathrm{mg} / \mathrm{kg})$. Total glutathione ( $\mathrm{tGSH})$, total oxidant (TOS), and total antioxidant (TAS) levels were tested together with aspartate aminotransferase (AST), alanine aminotransferase (ALT), lactate dehydrogenase (LDH) and malondialdehyde (MDA). Histopathological examination of the tissues was performed. We have found that levels of MDA, TOS, ALT, AST, and LDH were all higher in PP group than in HG and PT groups $(p<$ 0.05). In PP group, the TAS and tGSH levels were statistically substantially lower. The PT for oxidants levels showed a statistically significant reduction. In PT group, the levels of antioxidants were found to be considerably higher. The epitheliums, glands, and vascular structures of the PTs were histologically close to normal. By boosting antioxidants, TPP may help to reduce propofolinduced liver damage.
\end{abstract}

Key words: Antioxidants — Liver — Oxidative stress - Propofol — Thiamine pyrophosphate

\begin{abstract}
Abbreviations: ALT, alanine aminotransferase; AST, aspartate aminotransferase; GSH-Px, glutathione peroxidase; $\mathrm{LDH}$, lactate dehydrogenase; MDA, malondialdehyde; PIS, propofol infusion syndrome; PMNL, polymorpho-nuclear leukocytes; SOD, superoxide dismutase; TAS, total antioxidant; tGSH, total glutathione; TOS, total oxidant; TPP, thiamine pyrophosphate.
\end{abstract}

\footnotetext{
Correspondence to: Ufuk Kuyrukluyıldı, Department of Anesthesiology and Reanimation, Faculty of Medicine, Erzincan Binali Yildirim University, 24100, Erzincan, Turkey

E-mail: drufuk2001@gmail.com

(C) The Authors 2022. This is an open access article under the terms of the Creative Commons Attribution-NonCommercial 4.0 International License (https://creativecommons.org/licenses/by-nc/4.0/), which permits non-commercial use, distribution, and reproduction in any medium, provided the original work is properly cited.
} 


\section{Introduction}

Propofol (2,6-diisopropylphenol) is a common hypnotic agent used in many day surgeries and non-operating room anesthetic procedures since it has fewer adverse effects than other anesthetics (Uskur et al. 2021). Propofol, on the other hand, is regarded to be safe and possesses antiemetic, anti-pruritic, anxiolytic, bronchodilator, muscle relaxant, and anti-epileptic effects. However, its short-term high-dose or long-term low-dose usage has been linked to hepatotoxicity (Raisi et al. 2020). Prolonged administration of different doses of propofol for sedoanalgesia, especially in critically ill patients in reanimation units, may cause multi-organ and system damage known as the propofol infusion syndrome (PIS) (Hemphill et al. 2019). Animals that were given propofol suffered significant damage to their hearts, lungs, liver, gallbladder, kidneys, bladder, and skeletal muscles in experimental tests, and also multiple organ damage comparable to PIS in humans occurred (Ypsilantis et al. 2011). On the basis of the toxic impact of propofol, the research suggests that oxidative stress may be a significant component.

Although several clinical and experimental studies have indicated that propofol has an antioxidant effect (Marik 2004), other studies have also shown that short-term high-dose applications or long-term low-dose applications of propofol create an oxidant effect at the cell level, reducing the antioxidant effect (Yao and Zhang 2020). Propofol supplied at a dose of $50 \mathrm{mg} / \mathrm{kg}$ for a long period raised the level of malondialdehyde (MDA), which has an oxidant impact on muscle tissue, and lowered the amount of total glutathione (tGSH), which is an antioxidant, according to a recent experimental research. Furthermore, it was reported that administering twice the high dose of propofol raised the level of MDA in muscle tissue in a short period of time and decreased the amount of tGSH (Erdem et al. 2021).

We utilized thiamine pyrophosphate (TPP), an active metabolite of thiamine, to see if it might protect the propofol group from developing liver injury. TPP has been demonstrated in earlier research to reduce oxidative stress caused by alcohol and paracetamol in animal liver and to have a hepatoprotective effect by shielding the liver tissue from oxidative damage (Tung et al. 2005; Chin et al. 2008). TPP treatment also considerably reduces desflurane-related histopathological damage by boosting antioxidant activity (Arslan et al. 2016). We found no experimental trials examining the preventive effect of TPP against propofol-related hepatotoxicity in our assessment of the literature. As a result, we searched if TPP might protect the liver against potential propofol-induced liver oxidative damage.

\section{Materials and Methods}

\section{Animals}

18 albino male Wistar rats weighing 265-280 g were used in the study. Atatürk University's Medical Experimental Application and Research Center provided the animals. The animals were housed and fed in groups in regular laboratory circumstances prior to the study $\left(22^{\circ} \mathrm{C}\right)$. The Ataturk University's local animal ethics council in Erzurum, Turkey, approved the animal research, which followed the National Guidelines for the Use and Care of Laboratory Animals (Ethics Committee Number: 77040475-641.04-E.2100046921, Dated: 16.02.2021).

\section{Chemicals}

Fresenius Kabi Ilac San (Turkey; 1-20 ml ampule) provided propofol. IE Ulagay (Turkey) supplied the thiopental sodium. Biopharma (Russia) supplied the thiamine pyrophosphate.

\section{Experimental procedure}

The eighteen rats were split into three groups: HG, healthy; $\mathrm{PP}$, propofol-treated $(50 \mathrm{mg} / \mathrm{kg})$ and PT, treated with propofol $(50 \mathrm{mg} / \mathrm{kg})$ and TPP $(25 \mathrm{mg} / \mathrm{kg})$. To conduct the experiment, the PT $(n=6)$ group was administered $25 \mathrm{mg} / \mathrm{kg}$ TPP intraperitoneally (ip). At the same time, the PP $(n=6)$ and HG $(n=6)$ groups received an identical volume of distilled water ip $(0.5 \mathrm{ml})$. One hour after TPP and distilled water were administered, both groups received propofol intravenously at a dose of $100 \mathrm{mg} / \mathrm{kg}$. This procedure was repeated once a day for three days. Blood samples from all animal groups were analyzed for aspartate aminotransferase (AST), alanine aminotransferase (ALT), and lactate dehydrogenase (LDH) at the conclusion of the study. MDA, tGSH, total oxidant (TOS), and total antioxidant (TAS) levels were also measured from the tissue samples taken from the livers of animals killed with a high-dose anesthetic (thiopental sodium $50 \mathrm{mg} / \mathrm{kg}$ ). Some of the liver tissues were subjected to histopathological tests. All of the experimental groups' biochemical and histological data were compared and evaluated.

\section{Biochemical analyses}

\section{Biochemical analysis of liver tissue}

For biochemical studies of the tissues, homogenates of liver tissues were prepared. The levels of tGSH and MDA in the supernatants generated from these homogenates were determined using appropriate methods based on the literature. 
Preparation of the samples

0.2 grams of each tissue was extracted and weighed at this stage in the research. For tGSH measurement, $1.15 \%$ potassium chloride solution was added to $2 \mathrm{ml}$ of phosphate buffer with $\mathrm{pH}=7.5$ and homogenized in an iced condition. It was then centrifuged at $+4^{\circ} \mathrm{C}$ for $15 \mathrm{~min}$ at $10000 \mathrm{rpm}$. From the supernatant fraction, an analytical sample was obtained.

\section{Determination of $M D A$}

Spectrophotometric technique was used to measure the absorbance of the pink-colored complex formed by thiobarbituric acid (TBA) and MDA at a high temperature $\left(95^{\circ} \mathrm{C}\right)$ and wavelength of $532 \mathrm{~nm}$. To determine the amount of MDA in the supernatants, the homogenates were centrifuged at 5000 $\times g$ for $20 \mathrm{~min}$. $250 \mu \mathrm{l}$ of homogenate, $100 \mu \mathrm{l}$ of $8 \%$ sodium dodecyl sulfate (SDS), $750 \mu \mathrm{l}$ of $20 \%$ acetic acid, $750 \mu \mathrm{l}$ of $0.08 \% \mathrm{TBA}$, and $150 \mu \mathrm{l}$ of distilled water were pipetted and vortexed into sealed test tubes. After $60 \mathrm{~min}$ of incubation at $100^{\circ} \mathrm{C}, 2.5 \mathrm{ml}$ of $\mathrm{n}$-butanol was added, and the measurement was performed spectrophotometrically. The amount of MDA amount in the samples was estimated using a standard graph constructed using the previously made MDA stock solution and the dilution coefficients, and the quantity of red color produced was quantified at $532 \mathrm{~nm}$ using $3 \mathrm{ml}$ cuvettes (Ohkawa et al. 1979).

\section{Determination of $t G S H$}

Molecules with sulfhydryl groups quickly decreased the disulfide chromogen DTNB [5,5'-dithiobis (2-nitrobenzoic acid)] in the measurement solution. The resulting yellow hue was measured spectrophotometrically at $412 \mathrm{~nm}$. To determine the amount of GSH in the supernatants, the homogenates were centrifuged at $12000 \times g$ for $10 \mathrm{~min} .1500 \mu \mathrm{l}$ measurement buffer $(200 \mathrm{mM}$ Tris- $\mathrm{HCl}, 0.2 \mathrm{mM}$ EDTA, $\mathrm{pH}=8.2), 500 \mu \mathrm{l}$ supernatant, and $100 \mu \mathrm{l} 5.5$ '-dithio-bis (2-nitrobenzoic acid) (DTNB) were pipetted and vortexed into sealed test tubes with $7900 \mu \mathrm{l}$ methanol. A spectrophotometer was used to measure the mixture after $30 \mathrm{~min}$ of incubation at $37^{\circ} \mathrm{C}$. The amount of GSH in the samples was estimated using a standard graph established with the previously made GSH stock solution, considering the dilution factors, and the amount of yellow color produced was quantified at $412 \mathrm{~nm}$ using $3 \mathrm{ml}$ quartz cuvettes (Sedlak and Lindsay 1968).

\section{Measurements of TOS and TAS}

To measure the TOS and TAS levels in tissue homogenates, Erel developed a unique automated measuring method and produced commercially available kits (Rel Assay Diagnostics,
Turkey) (Erel 2004, 2005). Measurements were conducted at $660 \mathrm{~nm}$ using the TAS method, which depends on antioxidants bleaching the color of a more stable ABTS (2,2'-azinobis (3-ethylbenzothiazoline-6-sulfonic acid) radical cation. The results are reported in nmol per liter hydrogen peroxide $\left(\mathrm{H}_{2} \mathrm{O}_{2}\right)$ equivalents $\left(\mathrm{n} / \mathrm{mol} \mathrm{H}_{2} \mathrm{O}_{2}\right)$. In the TOS method, the oxidants in the sample oxidized the ferrous ion-o-dianisidine complex to ferric ion. The oxidation reaction was assisted by glycerol molecules, which are abundant in the reaction media. The ferric ion produced a colorful complex with xylenol orange in an acidic medium. The color intensity, which could be measured spectrophotometrically at $530 \mathrm{~nm}$, was related to the total amount of oxidant molecules present in the sample. The data are given in mol Trolox equivalents per liter. The percentage ratio of TOS to TAS was used to create the oxidative stress index (OSI). TOS was divided by $100 \times$ TAS to get the OSI.

\section{ALT analysis}

Using a Roche brand Cobas 8000 autoanalyzer, the spectrophotometric method was utilized to determine the quantitative measurement of serum ALT. ALT catalyzes the reaction between L-alanine and 2-oxoglutarate. NADH, giving $\mathrm{L}$-lactate and $\mathrm{NAD}^{+}$, in a process catalyzed by lactate dehydrogenase $(\mathrm{LDH})$, reduces the pyruvate produced. In the amino transfer process, pyridoxal phosphate functions as a coenzyme and guarantees that all enzymes are fully activated. L-alanine +2 -oxoglutarate $\rightarrow$ (ALT) pyruvate $+\mathrm{L}$-glutamate. The rate of oxidation of pyruvate $+\mathrm{NADH}$ $+\mathrm{H}^{+} \rightarrow(\mathrm{LDH})$ L-lactate $+\mathrm{NAD}+\mathrm{NADH}$ is directly proportional to the catalytic ALT activity.

\section{AST analysis}

Using a Roche brand cobas 8000 autoanalyzer, the spectrophotometric method was utilized to determine the quantitative measurement of serum AST. AST in the sample catalyzes the transfer of an amino group from L-aspartate to 2-oxoglutarate, resulting in oxaloacetate and L-glutamate. Oxaloacetate combines with $\mathrm{NADH}$ to generate NAD+ in the presence of malate dehydrogenase (MDH). In the amino transfer process, pyridoxal phosphate functions as a coenzyme. L-Aspartate + 2-oxoglutarate $\rightarrow$ (AST) oxaloacetate $+\mathrm{L}$-glutamate. Oxaloacetate $+\mathrm{NADH}+\mathrm{H}^{+} \rightarrow(\mathrm{MDH})$ $\mathrm{L}$-malate $+\mathrm{NAD}^{+}$. The rate of $\mathrm{NADH}$ oxidation is directly proportional to the catalytic AST activity.

\section{LDH analysis}

The quantitative analysis of serum LDH (P-L) was performed using a spectrophotometric method on a Roche brand cobas 8000 autoanalyzer. It is a conventional technique that has 
been optimized, according to the Deutsche Gesellschaft für Klinische Chemie (DGKC). LDH catalyzes the conversion of pyruvate to L-lactate and $\mathrm{NAD}^{+}$from pyruvate and $\mathrm{NADH}$. Pyruvate $+\mathrm{NADH}+\mathrm{H}^{+} \rightarrow(\mathrm{LDH})$ L-lactate $+\mathrm{NAD}^{+}$. The catalytic LDH activity is directly related to the initial rate of $\mathrm{NADH}$ oxidation. At a wavelength of $340 \mathrm{~nm}$, the reduction in absorbance was measured.

\section{Histopathological examination}

In a $10 \%$ formaldehyde solution, all of the tissue samples were examined under a microscope. After the identification procedure, the tissue samples were washed in cassettes with tap water for $24 \mathrm{~h}$. A normal grade of alcohol was then used to extract the water from the tissues (70, 80, 90 and 100\%). After passing through xylol, the tissues were preserved in paraffin. Four to five-micron slices cut from paraffin blocks were stained with hematoxylin and eosin, and their photos were taken during a test of the Olympus DP2-SAL firmware software (Olympus ${ }^{\oplus}$ Inc. Tokyo, Japan). A blinded pathologist carried out the research groups' histopathological evaluations. Each section's severity of histopathological findings was graded on a scale of 0 to 3 ( 0 , normal; 1 , mild injury; 2 , moderate injury; 3 , severe injury).

\section{Statistical analysis}

SPSS version 19 was used to conduct statistical analyses (IBM Corp. Released 2010 IBM SPSS Statistics for Windows, version 19 Armonk, NY: IBM Corp.). For each variable, descriptive statistics were computed. For continuous variables, the findings were reported as mean \pm standard deviation (SD). The one-way variance analysis (ANOVA) approach was used to evaluate the significance of the differences between the groups, followed by Tukey analysis. The statistical significance was defined to have a value of $p<0.05$.
The findings of the experiment were represented as mean \pm standard deviation (SEM). The one-way ANOVA test was used to evaluate the significance of the difference between groups. Then, Fisher's post hoc LSD (least significant differences) method was applied.

\section{Results}

When the MDA levels in the study groups were compared, it was shown that the PP group had greater levels than the HG group $(p<0.001)$. The MDA levels in the PT group were found to be lower than that in the PP group $(p<0.001)$ and similar to those in the HG group $(p=0.341)$. The tGSH levels in the PP group were found to be lower than that in the HG group $(p<0.001)$. The tGSH levels in the PT group, on the other hand, were found to be identical to those in the HG group, with no statistically significant difference $(p=0.082)$. The PP group's TOS levels were shown to be statistically significant $(p<0.001)$ when compared to the HG group. It was discovered that the PT group had similar levels of TOS as the HG group $(p=0.509)$. The reduction in TAS levels in the PP group was statistically significant $(p=$ 0.001) when compared to the HG group. When the PT group was compared to the HG group, there was no statistically significant difference $(p=0.105)$. When the ALT levels of the PP group were compared to the HG group, significant differences were detected $(p<0.001)$. The ALT level in the PT group was greater than that in the HG group $(p=0.008)$ but not statistically significant, whereas it was lower in the PP group $(p<0.001)$. When the AST levels were evaluated, the PP group had higher AST values; these differences were statistically significant $(p<0.001)$ when compared to the HG group. Despite being lower than the PP group $(p<$ $0.001)$, the AST value in the PT group was greater than that in the HG group $(p=0.001)$. The PP group's LDH level was

Table 1. Biochemical findings in study groups

\begin{tabular}{|c|c|c|c|c|c|c|c|}
\hline \multirow{2}{*}{ Parameter } & \multicolumn{3}{|c|}{ Group } & \multirow{2}{*}{$p$} & \multicolumn{3}{|c|}{ Pairwise comparisons $p$-values } \\
\hline & HG & PP & $\mathrm{PT}$ & & HG vs. PP & HG vs. PT & PP vs. PT \\
\hline MDA ( $\mu \mathrm{mol} / \mathrm{g}$ prot.) & $2.2 \pm 0.2$ & $4.7 \pm 0.4$ & $2.4 \pm 0.3$ & $<0.001$ & $<0.001$ & 0.341 & $<0.001$ \\
\hline tGSH (nmol/g prot.) & $5.2 \pm 0.4$ & $2.1 \pm 0.3$ & $4.7 \pm 0.4$ & $<0.001$ & $<0.001$ & 0.082 & $<0.001$ \\
\hline TOS (nmol $\mathrm{H}_{2} \mathrm{O}_{2} /$ mg prot.) & $6.3 \pm 0.2$ & $13 \pm 1.8$ & $7 \pm 0.6$ & $<0.001$ & $<0.001$ & 0.509 & $<0.001$ \\
\hline TAS ( $\mu$ molTroloxEquiv/mg prot.) & $8 \pm 0.3$ & $4.3 \pm 0.4$ & $7.5 \pm 0.4$ & $0.001^{*}$ & 0.001 & 0.105 & 0.035 \\
\hline ALT & $48.5 \pm 2.9$ & $155.8 \pm 7.1$ & $61 \pm 7.5$ & $<0.001$ & $<0.001$ & 0.008 & $<0.001$ \\
\hline AST & $40.8 \pm 1.8$ & $287.3 \pm 11.1$ & $59.3 \pm 5$ & $<0.001$ & $<0.001$ & 0.001 & $<0.001$ \\
\hline $\mathrm{LDH}$ & $140.3 \pm 10$ & $353.8 \pm 31.3$ & $169 \pm 8$ & $<0.001$ & $<0.001$ & 0.055 & $<0.001$ \\
\hline
\end{tabular}

${ }^{\star}$ Kruskal Wallis test was performed with Dunn test as post-hoc, otherwise one-way ANOVA was performed with Tukey HSD as post-hoc. Results were presented as mean \pm standard deviation. ALT, alanine aminotransferase; AST, aspartate aminotransferase; LDH, lactate dehydrogenase; MDA, malondialdehyde; TAS, total antioxidant; tGSH, total glutathione; TOS, total oxidant; TPP, thiamine pyrophosphate; $\mathrm{HG}$, healthy; PP, propofol-treated (50 mg/kg); PT, treated with propofol (50 mg/kg) and TPP ( $25 \mathrm{mg} / \mathrm{kg})$; prot., protein. 
Table 2. Histopathological examination findings in study groups

\begin{tabular}{|c|c|c|c|c|c|c|c|}
\hline & \multicolumn{3}{|c|}{ Group } & \multirow{2}{*}{$p$} & \multicolumn{3}{|c|}{ Pairwise comparisons $p$-values } \\
\hline & HG & $\mathrm{PP}$ & PT & & HG vs. PP & HG vs. PT & PP vs. PT \\
\hline Degeneration & $0.0(0.0-0.0)$ & $2.7(2.3-2.7)$ & $0.6(0.3-0.8)$ & $<0.001$ & $<0.001$ & 0.138 & 0.046 \\
\hline Congestion & $0.0(0.0-0.0)$ & $2.1(2.0-2.8)$ & $0.2(0.0-0.3)$ & 0.001 & 0.001 & 0.519 & 0.017 \\
\hline PMNL infiltration & $0.0(0.0-0.0)$ & $2.8(2.7-3.0)$ & $0.2(0.0-0.3)$ & 0.001 & $<0.001$ & 0.280 & 0.029 \\
\hline Pericellular edema & $0.0(0.0-0.0)$ & $2.7(2.7-2.8)$ & $0.3(0.2-0.5)$ & $<0.001$ & $<0.001$ & 0.134 & 0.045 \\
\hline Central vein diameter & $244(234-254)$ & $772(619-811)$ & $414(390-432)$ & 0.001 & $<0.001$ & 0.155 & 0.052 \\
\hline
\end{tabular}

Kruskal Wallis test was performed with Dunn test as post-hoc. Results were presented as median (minimum-maximum). PMNL, polymorpho-nuclear leukocytes. For more abbreviations, see Table 1.

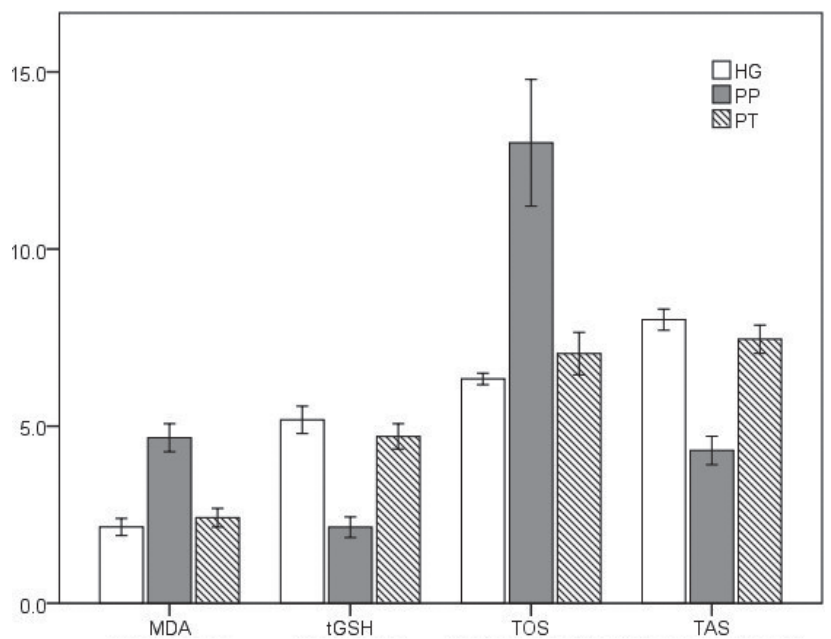

Figure 1. Malondialdehyde (MDA; $\mu \mathrm{mol} / \mathrm{g}$ prot.), total glutathione (tGSH; nmol/g prot.), total oxidant (TOS; nmol $\mathrm{H}_{2} \mathrm{O}_{2} / \mathrm{mg}$ prot.) and total antioxidant (TAS; $\mu \mathrm{mol}$ TroloxEquiv/mg prot.) levels in HG, PP and PT groups. HG, healthy; PP, propofol-treated (50 mg/ $\mathrm{kg})$; PT, treated with propofol $(50 \mathrm{mg} / \mathrm{kg})$ and thiamine pyrophosphate $(25 \mathrm{mg} / \mathrm{kg})$.

found to be statistically substantially greater than that of the HG group $(p<0.001)$. Although the LDH level in the PT group was significantly lower than that in the PP group $(p<0.001)$, it was comparable to the HG group $(p=0.055)$ (Table 1; Figs. 1 and 2).

\section{Histopathological evaluation}

In the HG group, the histological analysis of the liver tissue revealed that hepatocyte cell cords, Kupffer cells, cells, and vascular structures were all normal (Grade-0) liver tissue structures (Fig. 3A). When the PP group's liver tissue was analyzed, the nuclei and cell morphologies of the hepatocytes were found to be degenerated (Grade-3). While there was a lot of pericellular edema around the hepatocytes (Grade-3), there was also a lot of vasodilation and congestion in the central veins (Fig. 3A). Again, Grade-3 degraded hepatocyte nuclei and extremely significant polymorphonuclear cell infiltration in the parenchyma tissue were identified in the PP group's sections (Fig. 3B). This determinations' histopathological score was also shown to be statistically significant. When the treated PT group was examined, it was discovered that the hepatocytes and hepatocyte cell cords were in the normal structure, the central veins resembled the control group in appearance, and vasodilation and congestion had vanished in general (Fig. 3C). Histopathological regression was shown to be statistically significant using the grading method. Table 2 shows the results of the histopathological evaluations.

Degeneration was shown to be higher in the PP group than in the HG group $(p<0.001)$. The PT group had less degeneration than the PP group $(p=0.046)$ and was statistically equivalent to the healthy group $(p=0.138)$. The PP group had a statistically significant increase in congestion

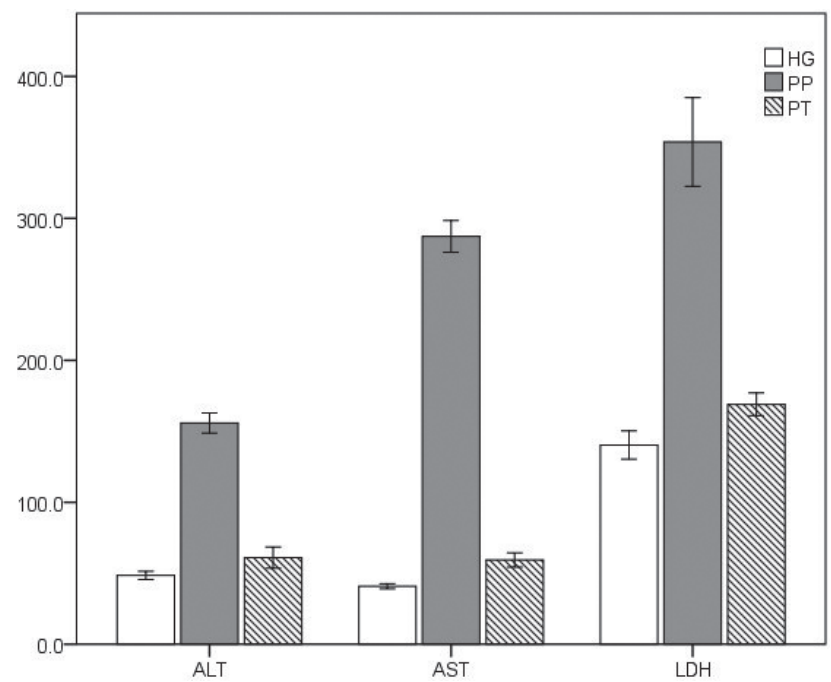

Figure 2. Alanine aminotransferase (ALT), aspartate aminotransferase (AST) and lactate dehydrogenase (LDH) levels in HG, PP and PT groups. For more abbreviations, see Fig. 1. 

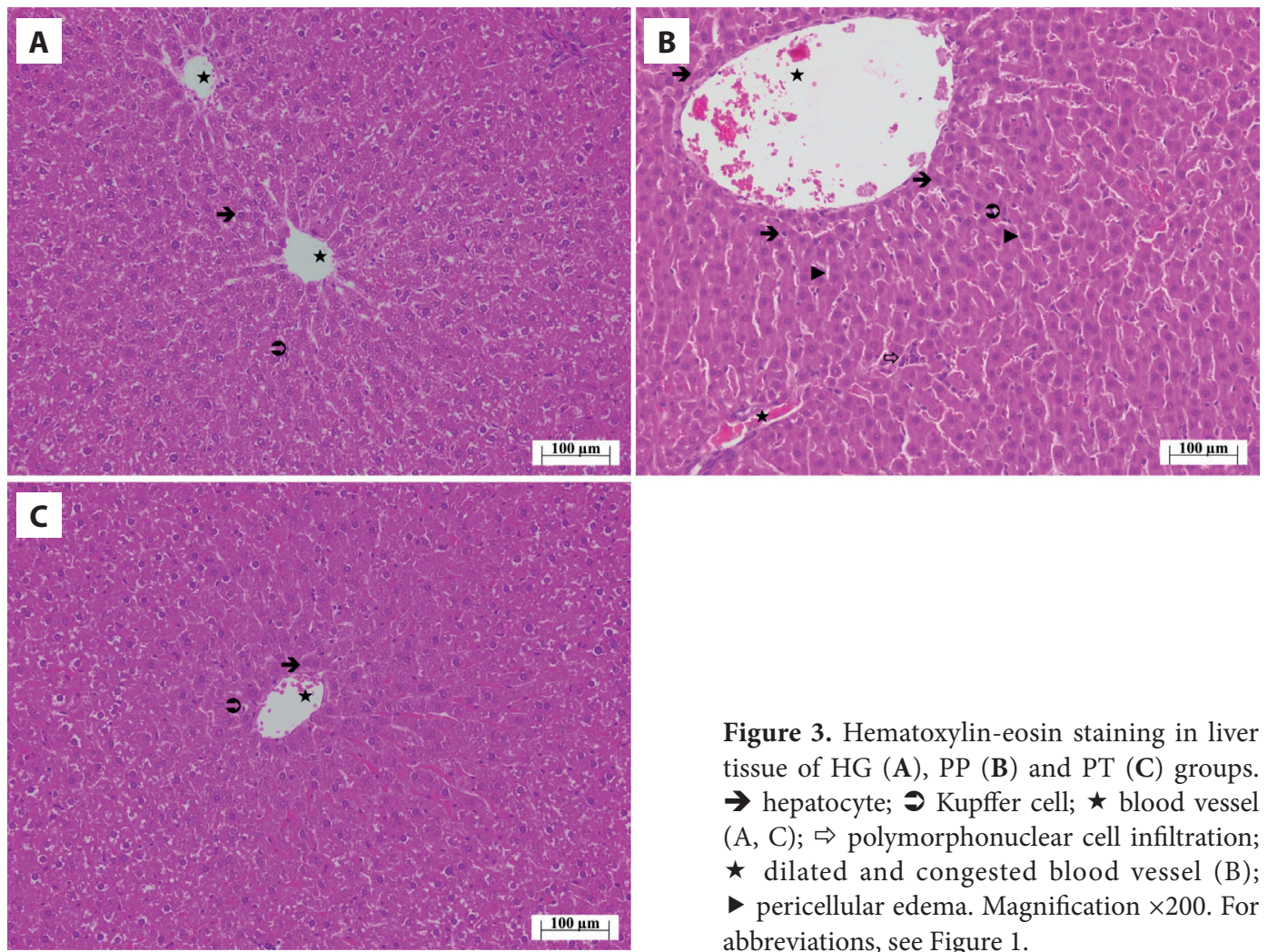

Figure 3. Hematoxylin-eosin staining in liver tissue of HG (A), PP (B) and PT (C) groups. $\rightarrow$ hepatocyte; $\rightarrow$ Kupffer cell; $\star$ blood vessel $(\mathrm{A}, \mathrm{C})$; $\Rightarrow$ polymorphonuclear cell infiltration; $\star$ dilated and congested blood vessel (B); - pericellular edema. Magnification $\times 200$. For abbreviations, see Figure 1 .

as compared to the HG group ( $p=0.001)$. The PT group had considerably less congestion $(p=0.017)$ than the PP group and was statistically equivalent to the HG group ( $p=$ 0.519). In comparison to the HG group, polymorphonuclear leukocyte (PMNL) infiltration was shown to be statistically substantially greater in the PP group $(p<0.001)$. The PMNL infiltration was found to be statistically substantially lower in the PT group than in the PP group $(p=0.029)$ and statistically similar in the HG group $(p=0.280)$. When comparing the PP and HG groups, pericellular edema was observed to be substantially greater in the PP group $(p<0.001)$. The PT group had statistically substantially less pericellular edema than the PP group $(p=0.045)$, whereas the HG group had no statistically significant difference $(p=0.134)$. The PP group had a significantly larger central vein diameter width than the HG group $(p<0.001)$. When comparing the PT group to the PP $(p=0.052)$ and HG $(p=0.155)$ groups, the central vein diameter was determined to be statistically similar (Table 2 ).

\section{Discussion}

Drug-induced liver damage is most common in the postoperative phase, and several anesthetic drugs are to blame. Propofol is a commonly used hypnotic agent in many non- operating room and day surgical procedures because it has fewer adverse effects than other anesthetic medications. Hypotension is the most common adverse effect, but pancreatitis, apnea, and acute hepatitis are all frequent. Propofol infusion syndrome, which develops after long-term propofol treatment, is frequently mentioned in the literature. Acute liver damage has also been reported in the postoperative phase in certain cases (Anand et al. 2001; Polo-Romero et al. 2008).

Biochemical tests such as ALT, AST, and LDH, as well as histological examination, have been utilized to detect drug-related acute liver damage in experimental studies published in the literature (Anand et al. 2001). As a result, in the methodology of our study, we employed ALT, AST, and $\mathrm{LDH}$ as biochemical markers to assess if propofolrelated liver damage exists. Biochemical parameters in the propofol group were found to be substantially higher than in the healthy group in our study. We discovered that the biochemical levels in the group that were given Thiamine pyrophosphate fell statistically substantially.

Ypislantis et al. (2007) discovered that the regions of inflammation and necrosis in the liver sections increased in the histological exams of the group that received propofol infusion for $24 \mathrm{~h}$. In the group that received $100 \mathrm{mg} / \mathrm{kg}$ propofol, the cell structure of the hepatocytes was disrupted, 
pericellular edema and significant dilatation was observed around the central veins, areas of both necrosis and inflammation were more common, and these changes were statistically significant, according to our findings. As a result, we interpreted the alterations we saw histopathologically in the propofol-administered group in our study as probable acute liver damage induced by propofol, as well as the elevations in biochemical markers.

There is some evidence that propofol causes liver damage due to its pathophysiology. Propofol has been shown to damage mitochondria, alter the formation and/or maintenance of the transmembrane electric potential (Branca et al. 1991), and disrupt electron transport throughout the mitochondrial electron chain in animal models (Schenkman and Yan 2000). Propofol is thought to influence fatty acid oxidation in humans by causing an increase in malonylcarnitine, which inhibits carnitine palmityl transferase 1, a mitochondrial long-chain fatty acid transport protein (Wolf et al. 2001). In our study, oxidation indicators such as MDA and TOS were found to be high in the propofol group, whereas antioxidant markers such as tGSH and TAS were found to be low. As a result, propofol was suspected of causing liver injury by increasing cellular oxidation.

Vitamin B complexes have been shown to have antioxidant effects (Depeint et al. 2006). TPP is the active form of the water-soluble vitamin thiamine. Thiamine pyrophosphokinase, an enzyme in the liver, produces it. Through the pentose phosphate pathway, it raises the antioxidant and NADPH levels (Turan et al. 2013). Thiamine, a nutrient that induces oxidative stress resistance, affects glucose metabolic control by shifting energy generation from fermentation to respiration (Kartal and Palabiyik 2019). Turan et al. (2014) utilized thiamine to reduce neurotoxicity caused by oxidative stress in the brain when combined with cisplatin.

In an experimental diabetic mellitus model, Sarandol et al. (2020) discovered that plasma and tissue MDA levels decreased in the thiamine-administered group compared to the control group, while antioxidant indicators such as superoxide dismutase (SOD) and glutathione peroxidase (GSH-Px) increased. Another research on thiamine found that thiamine deficiency raises the risk of oxidative damage (Hassan et al. 2020).

TPP was used in our study to prevent propofol-induced acute liver damage. The PP and PT groups had considerably reduced ALT, AST, and LDH values, which were used to diagnose liver injury. Furthermore, though the MDA and TOS levels were statistically substantially higher in the PP group, they were statistically significantly lower in the PT group. The antioxidant indicators such as tGSH and TAS, on the other hand, were shown to be statistically substantially greater in the PT group than in the PP group. These results were interpreted as indicating that TPP has antioxidant action in accordance with scientific literature and that propofol-related oxidative liver damage can be reduced by this impact.

Propofol is a commonly used anesthetic and intensive care medication, whose short-term high-dose usage, in particular, can induce oxidative damage to the liver and an increase in liver functions. Propofol-induced oxidative liver damage can be prevented by using TPP, an antioxidant medication.

As a result, we believe that TPP can be utilized to prevent propofol-induced acute liver damage, provided that clinical studies back up the findings of the trial.

Conflict of interest. The authors declare no conflict of interest.

Acknowledgments. The authors thank all physicians and other staff who contributed to the study.

\section{References}

Anand K, Ramsay MA, Crippin JS (2001): Hepatocellular injury following the administration of propofol. Anesthesiology 95, 1523-1524 https://doi.org/10.1097/00000542-200112000-00037

Arslan A, Kuyrukluyildiz U, Binici O, Cetin N, Balci MG, Kuzucu M, Yilmaz A, Altuner D, Coban TA (2016): Can thramıne pyrophosphate prevent desflurane induced hepatotoxicity in rats? Acta Cir. Bras. 31, 168-175

https://doi.org/10.1590/S0102-865020160030000004

Branca D, Roberti MS, Lorenzin P, Vincenti E, Scutari G (1991): Influence of the anesthetic 2,6-diisopropylphenol on the oxidative phosphorylation of isolated rat liver mitochondria. Biochem. Pharmacol. 42, 87-90 https://doi.org/10.1016/0006-2952(91)90684-W

Chin MW, Njoku DB, MacQuillan G, Cheng WS, Kontorinis N (2008): Desflurane-induced acute liver failure. Med. J. Aust. 189, 293-294

https://doi.org/10.5694/j.1326-5377.2008.tb02035.x

Depeint F, Bruce WR, Shangari N, Mehta R, O'Brien PJ (2006): Mitochondrial function and toxicity, role of the $\mathrm{B}$ vitamin family on mitochondrial energy metabolism. Chem. Biol. Interact. 163, 94-112 https://doi.org/10.1016/j.cbi.2006.04.014

Erel O (2004): A novel automated method to measure total antioxidant response against potent free radical reactions. Clin. Biochem. 37, 112-119 https://doi.org/10.1016/j.clinbiochem.2003.10.014

Erel O (2005): A new automated colorimetric method for measuring total oxidant status. Clin. Biochem. 38, 1103-1111 https://doi.org/10.1016/j.clinbiochem.2005.08.008

Erdem KTO, Bedir Z, Ates I, Kuyrukluyildiz U, Coban TA, Yazici GN, Arslan YK, Suleyman Z, Suleyman H (2021): The effect of adenosine triphosphate on propofol-induced myopathy in rats, a biochemical and histopathological evaluation. Korean J. Physiol. Pharmacol. 25, 69-77 https://doi.org/10.4196/kjpp.2021.25.1.69

Hassan MH, Awadalla EA, Ali RA, Fouad SS, Abdel-Kahaar E (2020): Thiamine deficiency and oxidative stress induced by 
prolonged metronidazole therapy can explain its side effects of neurotoxicity and infertility in experimental animals. Effect of grapefruit co-therapy. Hum. Exp. Toxicol. 39, 834-847 https://doi.org/10.1177/0960327119867755

Hemphill S, McMenamin L, Bellamy MC, Hopkins PM (2019): Propofol infusion syndrome, a structured literature review and analysis of published case reports. Br. J. Anaesth. 122, 448-459 https://doi.org/10.1016/j.bja.2018.12.025

Kartal B, Palabiyik B (2019): Thiamine leads to oxidative stress resistance via regulation of the glucose metabolism. Cell. Mol. Biol. 65, 73-77 https://doi.org/10.14715/cmb/2019.65.1.13

Marik PE (2004): Propofol, therapeutic indications and side-effects. Curr. Pharm. Des. 10, 3639-3649 https://doi.org/10.2174/1381612043382846

Ohkawa H, Ohishi N, Yagi K (1979): Assay for lipid peroxides in animal tissues by thiobarbituric acid reaction. Anal. Biochem. 95, 351-358 https://doi.org/10.1016/0003-2697(79)90738-3

Polo-Romero FJ, Paricio P, Tovar A, Alonso JM (2008): Propofolinduced acute toxic hepatitis after brief sedation for endoscopic retrograde cholangiopancreatography. Endoscopy 40, E49 https://doi.org/10.1055/s-2007-966881

Raisi H, Longerich T, Moreira Assuncao B, Mueller S, Schirmacher P, Seitz HK (2020): Propofol-induced hepatitis. Eur. J. Case Rep. Intern. Med. 7, 001921

Sarandol E, Tas S, Serdar Z, Dirican M (2020): Effects of thiamine treatment on oxidative stress in experimental diabetes. Bratisl. Lek. Listy 121, 235-2341 https://doi.org/10.4149/BLL_2020_036

Schenkman KA, Yan S (2000): Propofol impairment of mitochondrial respiration in isolated perfused guinea pig hearts determined by reflectance spectroscopy. Crit. Care Med. 28, 172-127 https://doi.org/10.1097/00003246-200001000-00028

Sedlak J, Lindsay RH (1968): Estimation of total, protein-bound, and nonprotein sulfhydryl groups in tissue with Ellman's reagent. Anal. Biochem. 25, 192-205 https://doi.org/10.1016/0003-2697(68)90092-4

Tung D, Yoshida EM, Wang CS, Steinbrecher UP (2005): Severe desflurane hepatotoxicity after colon surgery in an elderly patient. Can. J. Anaesth. 52, 133-136
https://doi.org/10.1007/BF03027717

Turan MI, Siltelioglu Turan I, Mammadov R, Altınkaynak K, Kisaoglu A (2013): The effect of thiamine and thiamine pyrophosphate on oxidative liver damage induced in rats with cisplatin. Biomed. Res. Int. 2013, 783809 https://doi.org/10.1155/2013/783809

Turan MI, Cayir A, Cetin N, Suleyman H, Siltelioglu Turan I, Tan H (2014): An investigation of the effect of thiamine pyrophosphate on cisplatin-induced oxidative stress and DNA damage in rat brain tissue compared with thiamine, thiamine and thiamine pyrophosphate effects on cisplatin neurotoxicity. Hum. Exp. Toxicol. 33, 14-21 https://doi.org/10.1177/0960327113485251

Uskur T, Şenöz AÖ, Çevreli B, Barlas A, Uzbay T (2021): Propofol but not dexmedetomidine produce locomotor sensitization via nitric oxide in rats. Psychopharmacology 238, 569-577 https://doi.org/10.1007/s00213-020-05707-5

Wolf A, Weir P, Segar P, Stone J, Shield J (2001): Impaired fatty acid oxidation in propofol infusion syndrome. Lancet 357 , 606-607 https://doi.org/10.1016/S0140-6736(00)04064-2

Yao Y, Zhang JJ (2020): Propofol induces oxidative stress and apoptosis in vitro via regulating miR-363-3p/CREB signalling axis. Cell Biochem. Funct. 38, 1119-1128 https://doi.org/10.1002/cbf.3572

Ypsilantis P, Politou M, Mikroulis D, Pitiakoudis M, Lambropoulou M, Tsigalou C, Didilis V, Bougioukas G, Papadopoulos N, Manolas C, Simopoulos C (2007): Organ toxicity and mortality in propofol-sedated rabbits under prolonged mechanical ventilation. Anesth. Analg. 105, 155-166 https://doi.org/10.1213/01.ane.0000265544.44948.0b

Ypsilantis P, Politou M, Mikroulis D, Lambropoulou M, Bougioukas I, Theodoridis G, Tsigalou C, Manolas C, Papadopoulos, Bougioukas G, Simopoulos C (2011): Attenuation of propofol tolerance conferred by remifentanil co-administration does not reduce propofol toxicity in rabbits under prolonged mechanical ventilation. J. Surg. Res. 168, 253-261 https://doi.org/10.1016/j.jss.2009.08.020

Received: September 7, 2021

Final version accepted: October 25, 2021 